\title{
TWO THEOREMS OF JOSEFSON-NISSENZWEIG TYPE FOR FRÉCHET SPACES
}

\author{
J. BONET, M. LINDSTRÖM, AND M. VALDIVIA \\ (Communicated by William J. Davis)
}

\begin{abstract}
We characterize the Fréchet-Montel (respectively, Fréchet-Schwartz) spaces by sequences in their dual spaces.
\end{abstract}

It is well known that a Fréchet space $E$ is Montel (respectively, Schwartz) if and only if it is separable and every weak*-null sequence in $E^{\prime}$ also converges strongly to zero (respectively, converges uniformly to zero on some zeroneighbourhood in $E$ ). Because of the Josefson-Nissenzweig theorem [3], Jarchow asked in [5] if these two results are true without the assumption of the separability condition on the Fréchet space $E$.

In this short note we give a positive answer to both of these questions. These results were discovered during the Conference of Function Theory on InfiniteDimensional Spaces held in the Universidad Complutense de Madrid from December 11-16, 1989. The Schwartz case had already been discovered independently by Lindström and Schlumprecht in [7] and by Bonet in [1].

We start by recalling the following result from [7], which is based on a very deep result due to Schlumprecht [8].

Theorem 1. Let $E$ be a Fréchet space such that every weak*-null sequence in $E^{\prime}$ is also strongly null-convergent. Then $E$ is reflexive.

Now we are ready to prove the announced results.

Theorem 2. A Fréchet space $E$ is Montel (respectively, Schwartz) if and only if weak*-null sequence in $E^{\prime}$ is also strongly null-convergent (respectively, converges uniformly to zero on some zero-neighbourhood in $E$ ).

Proof. Only the Montel case needs a proof because every Fréchet-Montel space is separable.

If $E$ is Montel then the condition is clearly necessary.

Let us now assume that every weak*-null sequence in $E^{\prime}$ is strongly nullconvergent. By the theorem above [7] $E$ must then be reflexive. Now we present two different proofs to show that $E$ is Montel.

First, by [6, Remark 1] it is enough to show that $U^{\circ}$ is $\sigma\left(E^{\prime}, E\right)$-sequentially compact for every zero-neighbourhood $U$ in $E$. To do this observe that $E_{\beta}^{\prime}$

Received by the editors January 25, 1991.

1991 Mathematics Subject Classification. Primary 46A04, 46A11. 
is a DF-space whose strong dual is $E$ by reflexivity. Hence $\sigma\left(E^{\prime},\left(E_{\beta}^{\prime}\right)^{\prime}\right)=$ $\sigma\left(E^{\prime}, E\right)$. Now according to [2, Theorem 11, Example 1.2(C)], every DF-space is weakly angelic (see, e.g., [4]), and since $U^{\circ}$ is always $\sigma\left(E^{\prime}, E\right)$-compact, it is also $\sigma\left(E^{\prime}, E\right)$-sequentially compact.

To give the second proof assume that $E$ is not Montel. Since $E$ is reflexive by the theorem above [7], there exists a weakly compact separable subset $A$ of $E$ that is not compact. Let $F$ be the closed linear span of $A$. By a recent result of Valdivia in [9], given $F$ we can find a closed separable subspace $G$ of $E$ that contains $F$ and has a topological complement $L$ in $E$. Certainly $G$ is separable but not Montel. Hence there is a $\sigma\left(G^{\prime}, G\right)$-null sequence in $L^{\perp}=G^{\prime}$ that is not $\beta\left(G^{\prime}, G\right)$-null convergent. Since $G$ is complemented in $E$, this contradicts the assumption on $E$.

\section{REFERENCES}

1. J. Bonet, A question of Valdivia on quasi-normable Fréchet spaces, Canad. Math. Bull. 34 (1991), 301-304.

2. B. Cascales and J. Orihuela, On compactness in locally convex spaces, Math. Z. 195 (1987), 365-381.

3. J. Diestel, Sequences and series in Banach spaces, Graduate Texts in Math., vol. 92, Springer, Berlin, Heidelberg and New York, 1984.

4. K. Floret, Weakly compact sets, Lecture Notes in Math., vol. 801, Springer-Verlag, New York, 1980.

5. H. Jarchow, Locally convex spaces, Teubner, Stuttgart, 1981.

6. M. Lindström and Th. Schlumprecht, On limitedness in locally convex spaces, Arch. Math. 53 (1989), 65-74.

7. __ A Josefson-Nissenzweig theorem for Fréchet spaces, Bull. London Math. Soc. (to appear).

8. Th. Schlumprecht, On dual spaces with bounded sequences without weak ${ }^{*}$ convergent convex blocs, Proc. Amer. Math. Soc. 107 (1989), 395-408.

9. M. Valdivia, Resolution of the identity operator in certain metrizable locally convex spaces, Rev. Real Acad. Ci. Madrid 83 (1989), 75-96.

Departamento de Matemática Aplicada, E.T.S. Arquitectura, Universidad Politécnica DE Valencia, E-46071 Valencia, Spain

E-mail address: JBONET@MAT.UPV.ES

Department of Mathematics, Abo Akademi, SF-20500 Abo, Finland

E-mail address: MLINDSTROM@FINABO.ABO.FI

Departamento de Análisis Matematico, Facultad de Matemáticas, Universidad de Valencia, 46100 Burjasot, Spain 